\title{
RADIATION DOSE TO THE EYE AND POTENTIAL OCCURRENCE RADIATION- INDUCED CATARACT FOLLOWING COMPUTED TOMOGRAPHY (CT) HEAD EXAMINATION
}

\author{
Muhammad Ikhmal Naim Mohd Hilal ${ }^{1}$, Rekha Ganesan ${ }^{1}$, Norhashimah Norsuddin ${ }^{1}$, Mohd Izuan Ibrahim ${ }^{1}$, \\ Said Mohd Shaffiq Said Rahmat ${ }^{2}$, Muhammad Khalis Abd Karim ${ }^{3}$ and Iza Nurzawani Che Isa ${ }^{1}$ \\ ${ }^{1}$ Diagnostic Imaging and Radiotherapy Programme, Faculty of Health Sciences, Universiti Kebangsaan \\ Malaysia, Jalan Raja Muda Abdul Aziz, 50300 Kuala Lumpur, Malaysia \\ 2Department of Radiology, National Cancer Institute, 4, Jalan P7, Presint 7, 62250 Putrajaya, Wilayah \\ Persekutuan Putrajaya, Malaysia \\ ${ }^{3}$ Department of Physics, Faculty of Sciences, Universiti Putra Malaysia, 43400 UPM Serdang, Selangor Darul \\ Ehsan, Malaysia
}

Corresponding author: Iza Nurzawani Che Isa

Email: zawaniisa@ukm.edu.my

\begin{abstract}
The eye is a radiosensitive organ that lies within the scan range during Computed Tomography (CT) of the head. The utilization of the head CT is increasing with growing concern about the chances of development of cataract which induces by ionising radiation. This research aimed to calculate eye absorbed dose and to study the potential occurrence of radiation induces cataracts between CT Brain and CT Temporal. A total of 399 set data were obtained retrospectively according to inclusion and exclusion criteria. 364 patients underwent CT Brain while 35 patients' data obtained for CT Temporal. The scanning parameters such as tube current, tube potential, pitch factor, beam width, filter, revolution time, and filter were recorded. Eye absorbed dose was significantly different $(p<0.05)$ between CT brain $(49.07 \pm 10.08 \mathrm{mGy})$ and CT temporal $(25.72 \pm 6.12 \mathrm{mG})$. None of the analysed data exceeded the eye threshold dose recommended by ICRP 2012. However, as expected, the cumulative eye absorbed dose was increased as the frequencies of the scan increase. The highest number of repeated scans is five times with cumulative dose was recorded as 278.27mGy. In conclusion, the eye absorbed dose is higher in CT Brain compared to CT Temporal and has potential for induction of cataract in the future especially with the patient that undergoes repeated CT examination.
\end{abstract}

Keywords: CT brain, CT temporal, cataract

\section{INTRODUCTION}

Computed Tomography (CT) was developed by Godfrey N. Hounsfield in 1973 with the idea to develop an algorithm of computer software that can compile X-ray beam from various angle of human anatomy and create a three-dimensional image that can assist the physician to visualize inside of the patient body ${ }^{1}$. The tremendous advancement in innovation and development of the multidetector CT (MDCT) scan has started in the late 1990s with 2-slices and continued to 640 slices makes CT scan is one of the important diagnostic modalities in healthcare institutions ${ }^{2}$. The fast acquisition and wider coverage in a single rotation that minimal table movement which able to reduce the scanning time, made it ideal for trauma, paediatric and geriatric scanning. These advantages also reduce the artefacts caused by movement and probability of repeated examination or need of sedation or general anaesthesia especially in children ${ }^{3}$. The Asia region reported as highest utilization of the $\mathrm{CT}$ scan for paediatric patients which is $9.6 \%$ in 2007 and $7.8 \%$ in 2009 that doubled than Europe. Head CT was recorded as the highest in the frequency of examination which $72 \%$ compared to other examinations ${ }^{4,5}$.
The indication for head CT is to evaluate any abnormality of the brain especially for trauma patients, detection of cancerous cells, study of the blood vessel, and brain-related disease. Highresolution CT (HRCT) is one of the primary techniques to assess temporal bone and osseous abnormality ${ }^{6}$. The dose delivered to the patient during CT scan examination is higher compared to conventional X-ray examination. The awareness about the radiation risk during $\mathrm{CT}$ scans among patients is unsatisfactory. The survey that has been conducted showed that only $34.19 \%$ of the patient aware that $\mathrm{CT}$ has a higher radiation dose as compared to conventional X-ray ${ }^{7}$. The patient that presents with recurrent or chronic disease will be indicated to undergoes continuous followup for CT examination are exposed to high radiation dose $e^{8,9}$. The risk and benefits of $\mathrm{X}$-ray imaging modality have become attention and critical observation in medical literature and health policy debate ${ }^{10}$.

The eyes are one of the most radiosensitive organs in the human body and will be unavoidably exposed to radiation within the scan range during head and temporal CT scans. The highest eye lens dose reported during standard brain protocol CT 
scan is about $61.0 \pm 3.1 \mathrm{mSv}$ that recorded in five hospitals in Johor Bharu, Malaysia and for CT Temporal was $40.17 \mathrm{mGy}$ of the routine helical $\operatorname{scan}^{6,11}$. This data may show that the eye received a high amount of radiation dose even it is not the indication of the examination. Radiogenic lens opacities are also known as cataract now known as the largest causes of visual impairment and blindness and it has been considered as deterministic effect with a threshold ${ }^{12}$. Current threshold data of radiation cataracts from Chernobyl clean workers and bomb survivors showed the dose threshold cortical opacities range about 0.1 to $0.7 \mathrm{~Gy}$ and may also associate with age, genetic and following exposure to radiation ${ }^{11,13}$. There are also few studies done to assess radiation cataracts among interventional staff and it is proved that posterior lens change is higher in cardiologists and nurses compared to the unexposed group. They concluded that low dose ionizing radiation harmful to the eye and the threshold value is maybe lower than the current guideline for radiation-induced cataracts. Based on the current publication from International Commission on Radiological Protection (ICRP), the threshold value for detectable opacities is currently within 0.5-2 Gy for acute exposure and $5 \mathrm{~Gy}$ for chronic exposure ${ }^{14}$. Hence, we develop the current study to evaluate patient eye lens dose during the brain and temporal CT examination and to estimate potential cataract occurrence following the head CT examination.

\section{METHODS}

\section{Study Design and Population}

This study was approved by the institutional ethics committee with the ethical code of UKM PPI/111/8/JEP-2019-147. A total of 399 data set that consist of Head and Temporal Computed Tomography (CT) were obtained retrospectively at the Radiology Department, Hospital Canselor Tuanku Muhriz (PPUKM), Cheras, Kuala Lumpur. The data was collected from January 2018 until December 2018. All patients were referred for plain head and temporal CT examination for a variety of clinical indications. The data selected including young adults aged between 18 to 39 years old who underwent plain head and temporal CT procedures by using a 640 slice CT machine (Aquilion One, Toshiba, Japan). Patient data of brain contrast study and incomplete scanning were excluded from the study.

\section{Data Extraction}

A 640 slice CT machine (Aquilion One, Toshiba, Japan) was installed at Radiology Department, PPUKM in 2017. All the images and parameter data are transferred automatically to the Freedom server after the examination was completed. All the parameter acquisition data was reviewed on Dicom Viewer (OsiriX MD, Pixmeo SARL, Geneva). A total of 399 patients were chosen as subjects in the current study. The data were distributed by 364 patients (224 male and 140 female) and 35 patients ( 22 male and 13 female) for CT Brain and CT Temporal examination, respectively. The total mean age of the subjects in the current study was $28 \pm 6$ years old. Tube potential was constant for most procedures at $120 \mathrm{kVp}$ and the mean of the tube current was recorded as $283.8 \pm 52.2 \mathrm{~mA}$. The starting point of most of the scanning was located at the vertex and glabella for CT Brain and CT Temporal, respectively. CT brain was proceeded using helical mode while for CT Temporal was acquired using 13 helical modes and 22 volumetric modes. Radiation exposure and acquisition parameters for both protocols were tabulated in Table 1.

Table 1: Radiation exposure and acquisition parameter for CT brain and CT temporal protocols

\begin{tabular}{lccccc}
\hline Protocol & $\begin{array}{c}\text { Tube } \\
\text { Potential } \\
(\mathrm{kVp})\end{array}$ & $\begin{array}{c}\text { Tube Current } \\
(\mathrm{mA})\end{array}$ & Pitch Factor & $\begin{array}{c}\text { Revolution } \\
\text { Time } \\
(\mathbf{s})\end{array}$ & $\begin{array}{c}\text { Scan Range } \\
(\mathrm{mm})\end{array}$ \\
\hline CT Brain & 120 & $280.35 \pm 52.03$ & $0.639 \pm 0.016$ & 0.75 & $162 \pm 20$ \\
CT Temporal & $118.0 \pm 4.71$ & $319.97 \pm 39.68$ & $0.863 \pm 0.175$ & $0.50 \pm 0.05$ & $117 \pm 17$ \\
\hline
\end{tabular}

\section{Dose Measurement and Potential Cataract Occurrence}

Organ dose for every acquisition was calculated by using a Web-based CT calculator (WAZA-ARIv2, Oita University of Nursing and Health Sciences \& JAEA, Japan). This software allowed automatic dose calculation based on the scanner model and by inserting all the acquisition parameters. This software also offers calculation of eye absorbed dose and other 27 organs, Dose Length Product (DLP), volume Computed

Tomography Dose Index (CTDIvol), and effective dose. Organ equivalent dose $(H T)(\mathrm{mSv})$ is given by:

$$
H_{T}=w_{R} \cdot D_{T \cdot R}
$$

where $w R$ is the radiation weighting factor ( $\mathrm{x}$ ray $=1$ ) and $D T . R$ indicates the absorbed dose to the organ $T$ from radiation $R$. Potential cataract occurrence was determined by referring to International Commission on Radiological Protection (ICRP) 2011 publication that mentioned about radiation-induced cataract threshold dose for acute exposure which is $0.5 \mathrm{~Gy}$.

\section{Data Analysis}

Statistical Packages for the Social Science (SPSS) version 23.0 window (SPSS Inc., USA) was used to 
analyse all the collected data. They were tabulated and presented in mean \pm standard deviation. All the data were collected and registered into the Microsoft Excel spreadsheet before transferred into SPSS for performing analysis. The independent samples T-test was used for comparison of the mean eye absorbed dose and potential cataract occurrence between patients that underwent brain and temporal CT examinations. Paired sample T-test was used to analyse the volume Computed Tomography Dose Index (CTDIvol) between CT console and WAZAARI software. The value $p<0.05$ was considered statistically significant differences.

\section{RESULTS}

\section{Radiation dose}

The comparison of the radiation dose between $\mathrm{CT}$ brain and CT temporal were presented in Table 2. The radiation dose for $\mathrm{CT}$ brain is greater as compared to temporal CT examinations. The eye absorbed dose between CT brain and CT temporal were significantly different with a $\mathrm{p}$-value less than 0.05 . The eye absorbed dose was recorded as $49.07 \pm 10.08 \mathrm{mGy}$ and $25.72 \pm 6.12 \mathrm{mGy}$ for CT brain and CT temporal, respectively. The maximum and minimum eye absorbed for $\mathrm{CT}$ brain were $11.49 \mathrm{mGy}$ and $66.69 \mathrm{mGy}$, respectively while for calculated, and compared between metaanalysis data from the CT console and Waza-Ari. There was a significant difference noted between these values $(p<0.05)$. The DLP per procedure between $C T$ brain and CT temporal were recorded as $1042 \pm 230 \mathrm{mGy} . \mathrm{cm}$ and $407 \pm 87 \mathrm{mGy} . \mathrm{cm}$, respectively, and were significantly different $(p<0.05)$.CT Temporal were $12.23 \mathrm{mGy}$ and 49.82 $\mathrm{mG}$, respectively. The effective dose for CT brain $(2.01 \pm 0.65 \mathrm{mSv})$ and $\mathrm{CT}$ temporal $(1.17 \pm$ $0.36 \mathrm{mSv}$ ) were significantly different with a $\mathrm{p}$ value less than 0.05 . The CTDIvol was measured,

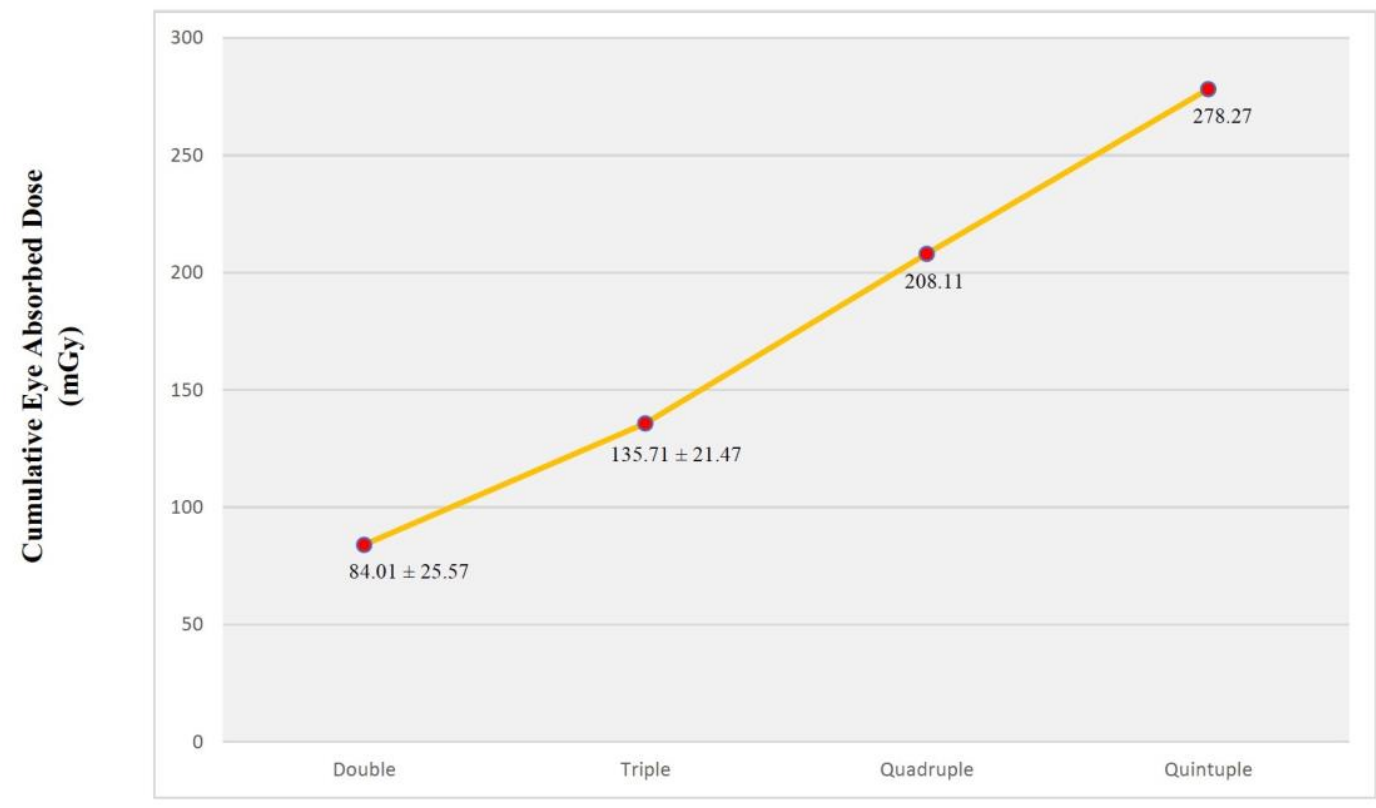

Exposure

FIGURE 1. The cumulative eye absorbed dose for multiple scanning

Table 2: Radiation dose for both CT brain and CT temporal protocols

\begin{tabular}{|c|c|c|c|c|c|c|}
\hline \multirow[t]{2}{*}{ Protocol } & \multicolumn{2}{|c|}{$\begin{array}{c}\text { Eye Absorbed Dose } \\
(\mathrm{mGy})\end{array}$} & \multicolumn{2}{|c|}{$\begin{array}{c}\begin{array}{c}\text { Effective Dose } \\
(\mathrm{mSv})\end{array} \\
\end{array}$} & \multicolumn{2}{|c|}{$\begin{array}{c}\text { Dose Length Product } \\
(\mathrm{mGy} . \mathrm{cm})\end{array}$} \\
\hline & Mean & Range & Mean & Range & Mean & Range \\
\hline CT Brain & $49.07 \pm 10.08$ & $11.49-66.69$ & $2.01 \pm 0.65$ & $0.49-7.38$ & $1042 \pm 330$ & $278-1894$ \\
\hline CT temporal & $25.72 \pm 6.12$ & $12.23-49.82$ & $1.17 \pm 0.36$ & $0.47-2.15$ & $407 \pm 87$ & $223-661$ \\
\hline
\end{tabular}

Note: All data was significantly different ( $p<0.05)$, range (maximum - minimum)

\section{Potential Occurrence of Cataract}

None of the acquisitions noted in the current study exceeded the threshold dose $(0.5 \mathrm{~Gy})$ to induce or detection of lens opacities for both CT Protocols under evaluation. Figure 1 showed the number of patients who underwent multiple CT scans within one year. It is noted that, as expected the mean of cumulative eye absorbed dose increased as the number of scans increased. The lowest dose was recorded in the patient who 
underwent two scans within one year $(84.01 \pm 25.57 \mathrm{mGy})$ while the highest number of scans recorded was five times (278.27 mGy).

\section{DISCUSSION}

The advancement of computed tomography technology has made it the primary diagnostic modality in healthcare facilities. Over the years, its utilization has significantly increased involving patients of a wide range of ages. Each CT exposes patients to the radiation dose that cumulatively could increase the biological risk, particularly in younger patients. The variation of the exposure factor as tabulated in Table 1, may contribute by patient size and the protocol that was selected during the acquisition. The differences in tube current and pitch factor may cause the variation of the DLP result. The patient physique could contribute to random variation while the various scan length and tube current may affect the radiation dose toward the patient ${ }^{15}$.

The eye is one of the radiosensitive organs that lie within the scan range even it is not a region of interest during $\mathrm{CT}$ brain and $\mathrm{CT}$ temporal examination. The mean eye absorbed dose for CT brain $(49.07 \pm 10.08 \mathrm{mGy})$ was higher as compared to CT temporal $(25.72 \pm 6.12 \mathrm{mGy})$. Table 2 showed variations of the eye absorbed dose noted between both protocols under investigation; CT brain and $\mathrm{CT}$ temporal. These variations may be contributed by the type of CT scanner, exposure factor, acquisition parameter, and technique to estimate absorbed dose. It is important to choose the appropriate technique in the estimation of the patient dose because it may affect the output data. Waza-ari is a web-based dosimetry system developed in Japan. Another crucial selection to be made is appropriate phantom size selection because it represents the population of the study. There were about $20 \%$ differences in organ dose noted between Japanese Phantom (JM) and ICRP phantom (16) due to the different sizes between both phantoms. It reflects Waza-ari ability to provide accurate organ dose according to the physical of Japanese patient and it is suitable for the Asian population ${ }^{16,17}$.

The different eye absorbed doses between both protocols may be caused by the location of the eye. During CT brain, eyes were located at the central portion of scanning compared to CT temporal that located near to starting point. The dose central dose is higher compared to the dose near the starting and endpoint of the scan along the $\mathrm{z}$-axis. This is because the scattered radiation is exposed toward the central portion of the scan by cranially and caudally outside of the beam width $^{18}$. Type of acquisition is one of the factors that lead to different doses between the brain and temporal CT examination. In this study, all CT brain was using helical mode scanning while $C T$ temporal were using helical and volumetric modes of scanning. The common problem related to helical scanning is over-beaming and over-ranging that may increase the radiation dose at the end and starting point of the scan. Kroft, Roelofs and Gelejins have proved via their study that volumetric mode scanning able to reduce dose $18 \%$ to $40 \%$ compared to helical mode scanning ${ }^{19}$.

A cataract is also known as one of the causes of blindness worldwide that can induce loss of lens clarity that may cause by ionizing radiation that leads to clouding and opacification of the eye $\mathrm{e}^{20}$. Foray et. $\mathrm{al}^{21}$ has discussed a different aspect of the individual response to radiation and classified cataract as a radio-degeneration defined as a noncancer effect. The conclusion was made from the publication of ICRP 2012 where the threshold dose has been changed from 5Gy to $0.5 \mathrm{~Gy}$ and was assumed that low radiation may produce lens opacities in $1 \%$ of the exposed individual after more than 20 years ${ }^{21}$.

No acquisitions in the current study recorded eye absorbed dose exceeded the threshold dose. However, as expected the number of cumulative eyes absorbed doses increased as the number of multiple scanning is increased. The patient that has chronic disease may have long-term follow-up which obliging them to undergoes repeated CT scans. Indirectly, they will be receiving high lens doses which have a positive correlation of increased risk of cataract ${ }^{9}$. The risk incidence of radiation-induced cataracts associated with an increasing number of CT exposure and potential occurrence of cataract is higher when cumulative CT exposure frequency more than four examinations ${ }^{22}$. For every patient that submitted for CT examination in the head region may increase the risk of cataract especially patient that undergoes multiple or repeated CT scan.

As we know, the eye lens is lies within the scanning range even it is not the region of interest of the examination. It is proven via several published data that the increase of the radiationinduced cataract during $C T$ head procedure ${ }^{8,21}$. The common scanning range for brain $\mathrm{CT}$ is from vertex until the base of the skull that starting from the infraorbitomeatal line (IOML). If the starting point of the scan were set superiorly, it may reduce the dose to the lens. About $11.8 \%$ $20.9 \%$ of the dose reduction was noted when the starting point was set from the superior orbital rim, instead of the inferior orbital rim $^{17}$. This proves that the eye lens reduces about $80 \%$ if the starting point of the scan was set superiorly. Gantry tilt and head tilt is a common alternative to moving the lens away from the scanning range as it can reduce absorbed dose by $75 \%$ and at the same time it able to reduce the scan length by few slices $^{23}$.

Three recommended techniques that can be used to reduce eye absorbed dose are bismuth eye shielding, organ-based Tube Current Modulation (TCM), and reduce tube current that can reduce 
up to $26.4 \%, 30.4 \%$, and $30.2 \%$, respectively. A combination of bismuth eye shielding and organbased TCM will reduce the dose around $40.7 \%$ from reference scan and this study concludes that reducing tube current around 30\% (from 250 to $177 \mathrm{mAs}$ ) can be achieved the same amount of eye dose reduction as bismuth shielding but in the same time can reduce other organ doses ${ }^{24}$. Niu et al. ${ }^{25}$ introduced modified helical mode scanning for temporal bone with scanning parameters like conventional helical scanning, no gantry angulation, and head in neutral position with scanning baseline parallel to the acanthiomeatal line. Modified helical scanning reduces the eye lens dose by $74.3 \%{ }^{25}$. Modifying scanning plane to head in neutral position with gantry tilting and scanning plane aligned to the acanthiomeatal line the eye dose reduce about $46.1 \%$ (from $35.63 \mathrm{mGy}$ to $19.20 \mathrm{mGy})^{26}$. Summary of reduction eye absorbed dose technique for CT Brain and CT Temporal among different author were shown in Table 3.
Standardization of the protocol is one of the main techniques that must be considered in every institution that involves the professional review of the physician and radiographer. According to Trattner et al. ${ }^{27}$, imaging physicians must review and create suitable CT protocol based on scan parameter, patient size and match with clinical indication, and may invite other specialists from different disciplines to review the suitability of the protocol. Diagnostic radiographer has to be included in the member of protocol review core team. Radiographers will perform the CT scan protocol, so they will see the bigger picture of the scanning session from the beginning until the end of the examination. They will see the workflow of the protocol and note any clinical implementation limitation and have to maintain and manage the protocol. The creation of the protocol has to achieve the goal to produce a good diagnostic image value based on the specific indication by using low dose rather than good quality of the image $^{27}$.

Table 3. Summary of dose reduction technique from different authors

\begin{tabular}{llc}
\hline \multicolumn{1}{c}{ Author } & \multicolumn{1}{c}{ Technique } & Dose Reduction \\
Kroff et al. (2010) & Volumetric acquisition mode & $18 \%$ to $40 \%$ \\
Suzuki et al. (2010) & Superior starting point & $80 \%$ \\
Futascsi (2014) \& & Gantry tilt & $75 \%$ \\
Nikupaavo et al. (2015) & & $26.4 \%$ \\
Wang et al. (2012) & Bismuth eye shielding & $30.4 \%$ \\
& Organ-based Tube Current Modulation & $30.2 \%$ \\
& (TCM) & $40.7 \%$ \\
Liu et al. (2010) & Combination bismuth eye shielding and & \\
Lee et al (2016) & organ-based TCM & $74.3 \%$ \\
\hline
\end{tabular}

\section{CONCLUSION}

In conclusion, there was a significant difference in eye absorbed dose and DLP between CT brain and CT temporal. In this study, no CT scan examination recorded exceeded the threshold dose to induce cataract for acute exposure. As expected, the cumulative eye absorbed dose increased as the number of repeated scanning increase. Radiographers, physicists, and radiologists have to collaborate in choosing and modify the best parameter and protocol so that we can reduce the absorbed dose for every patient. Even no data exceed the limit dose, the potential occurrence of radiation-induced cataract may happen in the future because the progression of cataract has a long latency period especially with the patient that undergoes multiple scanning. Finally, future consideration for next studies is to estimate the radiation dose following CT examination from the various hospital in Malaysia to evaluate in terms of acquisition parameter, radiation dose, and CT protocol.

\section{Conflict of interest}

The authors declare no potential conflict of interest.

\section{REFERENCES}

1. Bhattacharyya K. Godfrey Newbold Hounsfield (1919-2004): The man who revolutionized neuroimaging. Vol. 19, Annals of Indian Academy of Neurology. 2016. p. 448-50.

2. Edyvean S. CT equipment and performance issues: radiation protection 162. Radiat Prot Dosimetry 
[Internet]. 2013 Feb 1;153(2):190-6. Available from: https://doi.org/10.1093/rpd/ncs285

3. Rao P, Bekhit E, Ramanauskas F, Kumbla $\mathrm{S}$. $\mathrm{CT}$ head in children. Eur $\mathrm{J}$ Radiol. 2013;82(7):1050-8.

4. Vassileva J, Rehani MM, Al-Dhuhli $\mathrm{H}, \mathrm{Al}-$ Naemi HM, Al-Suwaidi JS, Appelgate K, et al. IAEA survey of pediatric CT practice in 40 countries in Asia, Europe, Latin America, and Africa: Part 1, frequency and appropriateness. Am J Roentgenol. 2012;198(5):1021-31.

5. Vassileva J, Rehani M, KostovaLefterova D, Al-Naemi HM, Al Suwaidi JS, Arandjic D, et al. A study to establish international diagnostic reference levels for paediatric computed tomography. Radiat Prot Dosimetry. 2015;165(14):70-80.

6. Thukral $C L$, Singh A, Singh $S$, Sood AS, Singh K. Role of High Resolution Computed Tomography in Evaluation of Pathologies of Temporal Bone. J Clin Diagn Res [Internet]. 2015 Sep;9(9):TC07-10. Available from: https: / /europepmc.org/articles/PMC46 06307

7. Singh N, Mohacsy A, Connell DA, Schneider ME. A snapshot of patients' awareness of radiation dose and risks associated with medical imaging examinations at an Australian radiology clinic. Radiography. 2017;23(2):94-102.

8. Alkhorayef $M$, Sulieman A, Alonazi $B$, Alnaaimi $M$, Alduaij $M$, Bradley $D$. Estimation of radiation-induced cataract and cancer risks during routine $\mathrm{CT}$ head procedures. Radiat Phys Chem. 2019;155:65-8.

9. Michel M, Jacob S, Roger G, Pelosse B, Laurier $D$, Le Pointe $H D$, et al. Eye lens radiation exposure and repeated head CT scans: A problem to keep in mind. Eur J Radiol [Internet]. 2012;81(8):1896900. Available from: https://www.sciencedirect.com/scienc e/article/pii/S0720048X11003214

10. Elmoheen A, Salem W, Bashir K. Reducing unnecessary $\mathrm{CT}$ scan of the head for minor paediatric head injuries at the emergency department. BMJ Open Qual. 2021;10(1).

11. Karim MKA, Hashim $S$, Sabarudin A, Bradley DA, Bahruddin NA. Evaluating organ dose and radiation risk of routine
CT examinations in Johor Malaysia. Sains Malaysiana. 2016;45(4):567-73.

12. Rehani MM, Vano E, Ciraj-Bjelac O, Kleiman NJ. Radiation and cataract. Vol. 147, Radiation Protection Dosimetry. 2011. p. 300-4.

13. Görtz H, Al Halabi A. The radiation cataract-an actual risk for vascular surgeons. Vol. 25, Gefasschirurgie. 2020. p. 6-11.

14. Clement CH, Stewart FA, Akleyev A V., Hauer-Jensen $M$, Hendry JH, Kleiman $\mathrm{NJ}$, et al. ICRP publication 118: ICRP Statement on Tissue Reactions and Early and Late Effects of Radiation in Normal Tissues and Organs Tissue Reactions in a Radiation Protection Context. Ann ICRP. 2012;41(1-2):1-322.

15. Litmanovich DE, Tack DM, Shahrzad M, Bankier AA. Dose Reduction in Cardiothoracic CT: Review of Currently Available Methods. RadioGraphics [Internet]. 2014 Oct 1;34(6):1469-89. Available from: https://doi.org/10.1148/rg.346140084

16. Ban N, Takahashi F, Sato K, Endo A, Ono $\mathrm{K}$, Hasegawa T, et al. Development of a web-based CT dose calculator: WazaARI. Radiat Prot Dosimetry. 2011;147(12):333-7.

17. Takahashi $F$, Sato K, Endo A, Ono K, Ban $\mathrm{N}$, Hasegawa $\mathrm{T}$, et al. Numerical analysis of organ doses delivered during computed tomography examinations using Japanese Adult Phantoms with the WAZA-ARI dosimetry system. Health Phys. 2015;109(2):104-12.

18. Suzuki $\mathrm{S}$, Furui $\mathrm{S}$, Ishitake $\mathrm{T}$, Abe $\mathrm{T}$, Machida $H$, Takei $R$, et al. Lens exposure during brain scans using multidetector row CT scanners: Methods for estimation of lens dose. Am J Neuroradiol. 2010;31(5):822-6.

19. Kroft LJM, Roelofs JJH, Geleijns J. Scan time and patient dose for thoracic imaging in neonates and small children using axial volumetric 320 -detector row CT compared to helical 64-, 32-, and 16detector row CT acquisitions. Pediatr Radiol. 2010;40(3):294-300.

20. Dauer LT, Ainsbury EA, Dynlacht J, Hoel $D$, Klein BEK, Mayer D, et al. Guidance on radiation dose limits for the lens of the eye: Overview of the recommendations in NCRP commentary 
no. 26. Vol. 93, International Journal of Radiation Biology. 2017. p. 1015-23.

21. Foray $\mathrm{N}$, Bourguignon $M$, Hamada $N$. Individual response to ionizing radiation. Mutat Res - Rev Mutat Res. 2016;770:369-86.

22. Yuan MK, Tsai DC, Chang SC, Yuan MC, Chang SJ, Chen HW, et al. The risk of cataract associated with repeated head and neck CT studies: A nationwide population-based study. Am J Roentgenol. 2013;201(3):626-30.

23. Nikupaavo U, Kaasalainen $\mathrm{T}$, Reijonen $\mathrm{V}$, Ahonen SM, Kortesniemi M. Lens dose in routine head CT: Comparison of different optimization methods with anthropomorphic phantoms. Am J Roentgenol. 2015;204(1):117-23.

24. Wang J, Duan X, Christner JA, Leng S, Grant KL, McCollough CH. Bismuth shielding, organ-based tube current modulation, and global reduction of tube current for dose reduction to the eye at head CT. Radiology. 2012;262(1):191-8.

25. Niu Y, Wang Z, Liu Y, Liu Z, Yao V. Radiation dose to the lens using different temporal bone $\mathrm{CT}$ scanning protocols. Am J Neuroradiol. 2010;31(2):226-9.

26. Lee TF, Chu CY, Sung HT, Leung ST, Leung KW, Kan WK. Reduction in lens Dose in temporal Bone Computed tomography by Modification of Scanning Plane. Hong Kong J Radiol. 2016;19(2):91-5.

27. Trattner S, Pearson GDN, Chin C, Cody DD, Gupta R, Hess CP, et al. Standardization and optimization of CT protocols to achieve low dose. J Am Coll Radiol. 2014;11(3):271-8. 